\title{
Mosses of Peatlands in Isla Grande de Chiloé - Chile: keys for identification
}

\section{Musgos de turberas en la Isla Grande de Chiloé - Chile: claves para identificación}

\author{
Carolina A. León ${ }^{1 *}$, Juan Larraín² \& Gisela Oliván-Martínez ${ }^{3}$ \\ 1Universidad Bernardo O’Higgins, Centro de Investigación en Recursos Naturales y Sustentabilidad, Fábrica 1990, segundo \\ piso, Santiago, Chile. \\ ${ }^{2}$ Instituto de Biología, Facultad de Ciencias, Pontificia Universidad Católica de Valparaíso, Campus Curauma, Av. Universidad \\ 330, Curauma, Valparaíso, Chile. \\ ${ }^{3}$ Departamento de Biología Vegetal I, Facultad de Ciencias Biológicas, Universidad Complutense de Madrid, E-28040 Madrid. \\ *carolina.leon@ubo.cl
}

\begin{abstract}
RESUMEN
Los briófitos juegan un rol clave en las turberas. Sin embargo, el conocimiento de este grupo es escaso en el sur de Sudamérica. Se presenta una clave de identificación de musgos de turberas de la Isla Grande de Chiloé (Chile). La clave está separada para cada grupo con caracteres similares.
\end{abstract}

\section{INTRODUCTION}

Mosses dominate the ground layer in southern Chilean peatlands. The function of the peatland ecosystem is highly dependent on this moss layer, and both production and decomposition, as well as community development, are all influenced by this layer of mosses (Vitt \& Wieder 2008). Unfortunately, we know little about this component of the flora, especially in southern South America.

In Chile, knowledge of mosses is scarce. There is still lacking a national compilation of the moss flora. There are some monographic works, but they only include a few orders and families. Moreover, there are only a few works that include keys for determination of species, like the mosses of the Juan Fernández Archipelago (Robinson 1975), the mosses of Senda Darwin Biological Station in Chiloé (Larraín 2007), or the mosses of Isla Navarino (Buck \& Goffinet 2010).

For this reason, the aim of this work is to present a key for determination of mosses focused in peatland habitats of Isla Grande de Chiloé in southern Chile $\left(41^{\circ}-43^{\circ} \mathrm{S}, 75^{\circ}\right.$ $73^{\circ} \mathrm{W}$ ). This study will contribute to increase the knowledge of the local bryophyte flora and facilitate the identification of these organisms.

\section{STUDY AREA}

Isla Grande de Chiloé is located in the Los Lagos Region of Chile $\left(42^{\circ}-43^{\circ} \mathrm{S}\right.$ and $\left.73^{\circ}-75^{\circ} \mathrm{W}\right)$. Chiloé's climate is wet temperate with a strong oceanic influence (Di Castri \& Hajek 1976). Mean summer temperature is $10.2{ }^{\circ} \mathrm{C}$ and mean winter temperature is $6.2{ }^{\circ} \mathrm{C}$ (Pérez et al. 2003). Annual rainfall fluctuates between 1,900 and 2,300 mm (CONAF 2009).

We studied mosses of peatlands dominated by lax cushions of Sphagnum moss associated with other bryophytes, lichens, sedges, rushes, and elfin shrubs, as well as swampy shrubland habitats dominated also by Sphagnum (Fig. 1).

\section{RESULTS}

Forty-four species in 29 genera are included. Campylopus, Dicranoloma and Sphagnum are the most species-rich genera and the best-represented families are Sphagnaceae and Dicranaceae. This key is based mainly on vegetative characters, when possible. A separate key for each group with similar traits, and an alphabetical list of taxa are provided. 


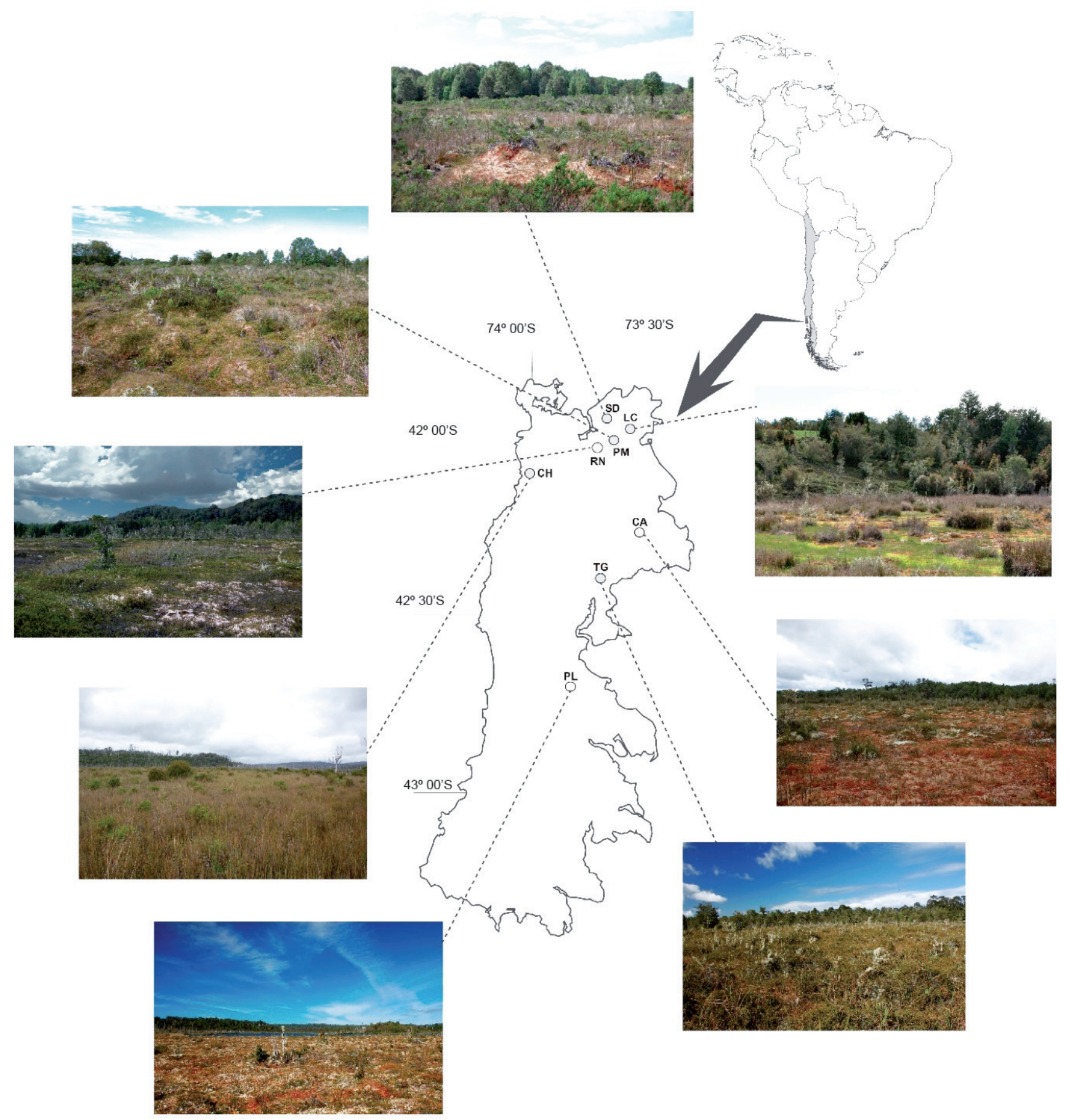

Figura 1. Location of studied peatlands in Chiloé island. PL, Púlpito; CA, Caulles; and RN, Río Negro; SD, Senda Darwin; CH, Chepu; PM, Pumanzano; LC, Lecam; and TG, Teguel. / Localidades de las turberas estudiadas en la isla de Chiloé. PL, Púlpito; CA, Caulles; and RN, Río Negro; SD, Senda Darwin; CH, Chepu; PM, Pumanzano; LC, Lecam; and TG, Teguel. 
1. Plants with branches in fascicles; laminal cells dimorphic, leaf lamina consisting of narrow green cells in a network enclosing large, inflated hyaline cells Sphagnum (Group A)

1'. Plants lacking the above combination of characters

...........................2

2 . Leaves with lamellae on the ventral surface Polytrichum longisetum

2'. Leaves without lamellae ....

3. Leaf cell walls strongly sinuous and nodulose

3'. Leaf cells not as above

4. Stems erect, simple or sparsely branched, forming mats or cushions; sporophytes terminal (on occasions apparently lateral due to subapical shoots); plants acrocarpous

4'. Stems usually prostrate, creeping and freely branched, forming carpets; sporophytes lateral, arising from a perichaetial bud or a short specialized branch; plants pleurocarpous

5. Leaves with narrow costa, absent in the upper third, sometimes completely absent; capsule with red annulus and peristome .....

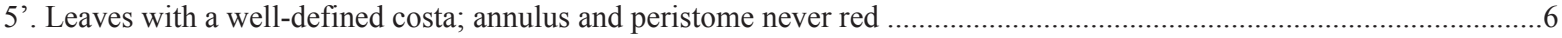

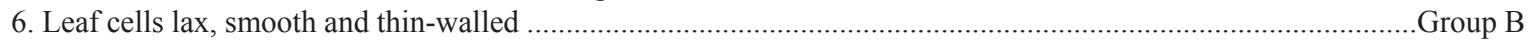

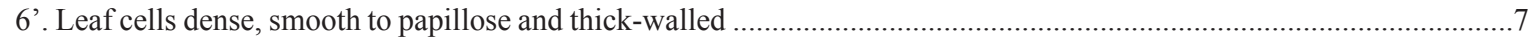

7. Costa broad (more than $1 / 3$ the width at the base of leaf), filling the leaf apex ..............................................oup C

7'. Costa narrow (less than $1 / 3$ the width at the base of leaf), not filling the leaf apex .............................................

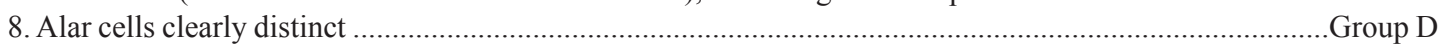

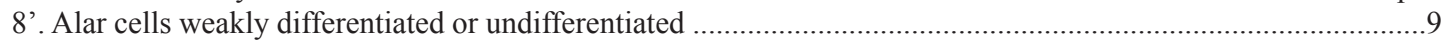

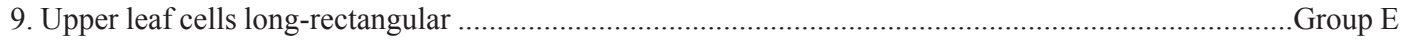

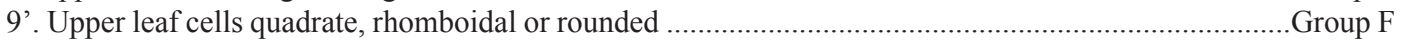

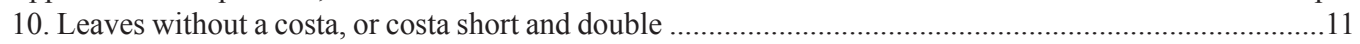

10 '. Leaves with a well-defined single costa reaching at least midleaf ................................................... 12

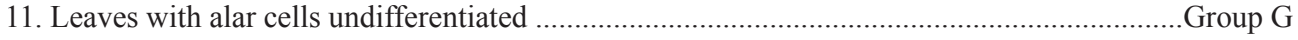

11 '. Leaves with alar cells distinct ..........................................................................................oup $\mathrm{H}$

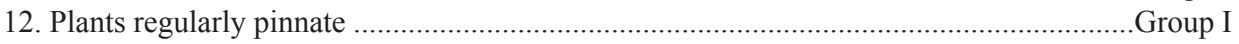

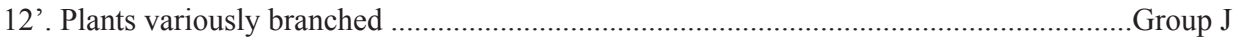

\section{GROUP A. Sphagnum}

1. Cortical cells of stem and branches usually with spiral fibrils; branch leaf green cells in cross-section rounded, centrally located, completely enclosed by adjacent hyaline cells

1'. Cortical cells of stem and branches without spiral fibrils; branch leaf green cells not as above

Sphagnum magellanicum

2. Branch leaf green cells in cross-section oval with equal exposure on both surfaces; stem hyalodermis in 1 layer; hyaline cells of branch leave with numerous pores crowded in bead-like rows along the commissures; brownish green plants ..........Sphagnum subsecundum 2'. Branch leaf green cells in cross-section triangular with unequal exposure towards one side; pores few to numerous, not placed along the hyaline cells commissures; plants bright green, yellow or reddish .....

3. Branch leaf green cells in cross-section triangular with more exposure on the outer surface; yellowish green plants, usually submerged.....

3'. Branch leaf green cells in cross-section triangular with more exposure on the inner surface .......

4. Branch leaves strongly crisped and squarrose when dry

4'. Branch leaves straight when dry

5. Stem leaves spathulate, fimbriate around the whole upper part; plants yellowish-green

5'. Stem leaves triangular with acute apex; plants pink or bright red

GROUP B. Acrocarpous. Leaf cells lax, smooth and thin-walled.

1. Leaves distinctly bordered; costa excurrent

Bryum pseudotriquetrum

1 '. Leaves not bordered; costa percurrent

Pohlia nutans

\section{GROUP C. Acrocarpous. Leaf cells dense and thick-walled, costa broad.}

1. Costa cells homogeneous in cross-section; capsule pyriform; peristome double

1'. Costa cells heterogeneous in cross-section; capsule ovoid to oblong-cylindrical; peristome single

2. Leaf cross-section with a clear medial row of guide cells between a ventral and a dorsal layer of stereids ....Chorisodontium aciphyllum

2'. Leaf cross-section not as above 3 (Campylopus)

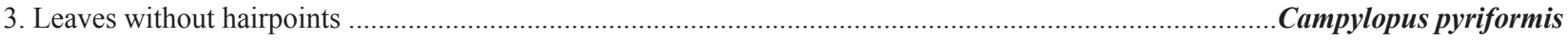

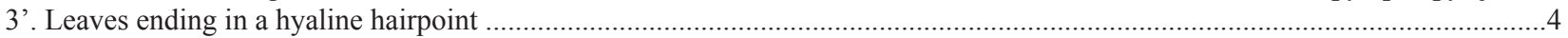

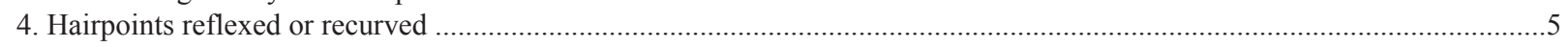

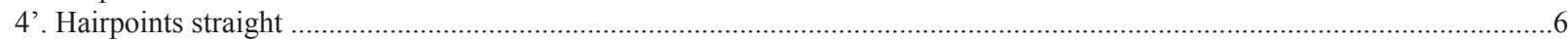

5. Hairpoints reflexed; costa often with dorsal lamellae to 2 cells tall ....................................................mpylopus introflexus

5'. Hairpoints recurved less than $90^{\circ}$; costa without dorsal lamellae.....................Campylopus aureonitens subsp. recurvifolius 
7. Transverse section of costa with ventral stereids; costa filling more than $1 / 2$ of leaf width at base; slender plants, usually light green Campylopus clavatus 7'. Transverse section of costa with ventral hyalocysts; costa filling less than $1 / 2$ of leaf width at base; plants robust, usually black or brown

Campylopus acuminatus

GROUP D. Acrocarpous. Leaf cells dense; costa narrow and alar cells differentiated.

1. Leaves lanceolate with long acumen (equal or longer than lamina); costa with more than 6 guide cells in cross-section

1'. Leaves lanceolate with short acumen; costa with 2-5 guide cells in cross-section

Dicranoloma robustum

2. Leaves falcate, ended in a flat and serrate apex; plants green to yellow-green

Dicranoloma billardierei

2'. Leaves straight, ending in an entire and channelled apex; plants bright yellow

.Dicranoloma imponens

GROUP E. Acrocarpous. Leaf cells dense; costa narrow; alar cells weakly differentiated or undifferentiated and upper leaf cells elongate-rectangular.

1. Leaf base not bordered; leaves plicate at base only

Breutelia dumosa

1 '. Leaf base bordered by narrow and elongate cells; leaves plicate throughout Breutelia subplicata

GROUP F. Acrocarpous. Leaf cells dense; costa narrow; alar cells weakly differentiated or undifferentiated and upper leaf cells quadrate, rhomboidal or rounded.

1. Leaves 5-ranked

1 '. Leaves not conspicuously ranked

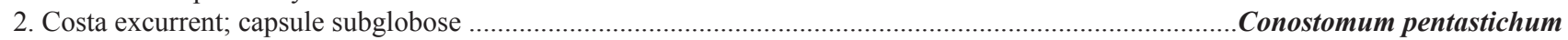

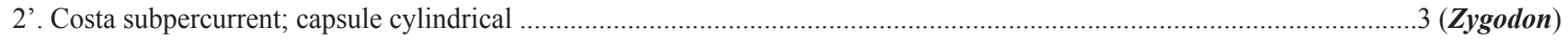
3. Stems prostate with erect branches; leaves more than $2 \mathrm{~mm}$ long, clearly 5-ranked; basal leaf cells dimorphic in definite yelloworange bands alternating with hyaline bands

3' Stems erect; leaves diffusely 5-ranked, less than $1 \mathrm{~mm}$ long; basal leaf cells without alternating color bands

Zygodon pentastichus

Zygodon hookeri var. leptobolax

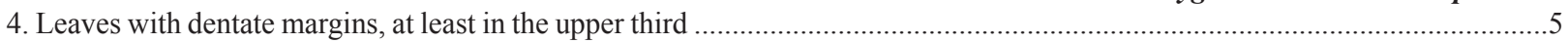

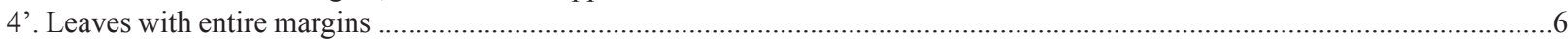

5. Leaf margin bistratose with double teeth; costa with dorsal spines; leaf cells smooth ....................Hymenodontopsis mnioides

5'. Leaf margin unistratose with single teeth; costa smooth; leaf cells papillose .....

Leptodontium longicaule var. microruncinatum

6. Marginal cells at leaf base with thickened transverse walls; leaves without sheathing base, straight; plants epiphytic ....

Ulota rufula

6'. Marginal leaf cells with transverse and longitudinal walls evenly thickened; leaves subulate with a sheathing base, strongly falcate to circinate; plants terrestrial, often submerged

Dicranella circinata

GROUP G. Pleurocarpous. Costa weak or absent. Alar cells undifferentiated

1. Costa single, weak, vanishing below midleaf; capsules smooth

Lepyrodon patagonicus

1 '. Costa double and short or absent; capsules ribbed

2. Leaves small (1.0-2.5 mm long), loosely inserted, spreading and clearly longitudinally plicate .................Ptychomniella ptychocarpa

2. Leaves medium or large $(2.0-6.8 \mathrm{~mm})$, densely imbricate, squarrose or recurved and plicate only at base

3. Leaves medium sized $(2.0-4.1 \mathrm{~mm})$, strongly squarrose; leaf apex abruptly acuminate ......

3'. Leaves large (4.0-6.8 $\mathrm{mm})$, straight to slightly recurved; leaf apex gradually acuminate

Ptychomnion densifolium .Ptychomnion cygnisetum

GROUP H. Pleurocarpous. Costa not conspicuous. Alar cells differentiated

1. Alar cells weakly differentiated from surrounding cells

1'. Alar cells strongly differentiated from surrounding cells

2. Stems with central strand; pseudoparaphyllia narrow-lanceolate to subfilamentose; flagelliform branches usually present; plants terricolous Hypnum cupressiforme var. mossmanianum 2'. Stems lacking central strand; pseudoparaphyllia more broadly foliose; flagelliform branches lacking; plants epiphytic

Hypnum chrysogaster 3. Leaves narrowly lanceolate, falcate, gradually narrowed into a long slender acumen .....................Rhaphidorrhynchium callidum

3'. Leaves broadly ovate-oblong to elliptical, straight, apex obtuse or rounded

4. Stem leaves oblong to cordate-ovate; alar cells small, forming a well-defined, auriculate, excavate, and rounded to broadly ovate group ............................................................................................................................Acrocladium auriculatum 4'. Stem leaves oblong-ovate; alar cells large, hyaline and inflated, not excavate, forming a triangular or \pm quadrate group

Calliergonella cuspidata 
GROUP I. Pleurocarpous. Costa conspicuous. Plants regularly pinnate.

1. Leaves dimorphic, stem leaves wide-cordate and branch leaves linear-lanceolate

1 '. Leaves monomorphic

Thuidiopsis furfurosa

2. Stems with numerous paraphyllia; leaf cells papillose Rigodium pseudothuidium

2'. Stems without paraphyllia; leaf cells smooth

Group J. Pleurocarpous. Costa conspicuous. Plants variously branched.

1. Leaves strongly falcate-secund, longitudinally plicate; alar cells forming a clear triangular group; leaf marginal cells not porose; seta smooth throughout

Sanionia uncinata

1'. Leaves straight, smooth; alar cells not differentiated; leaf marginal cells with porose walls; seta rough distally

Daltonia gracilis

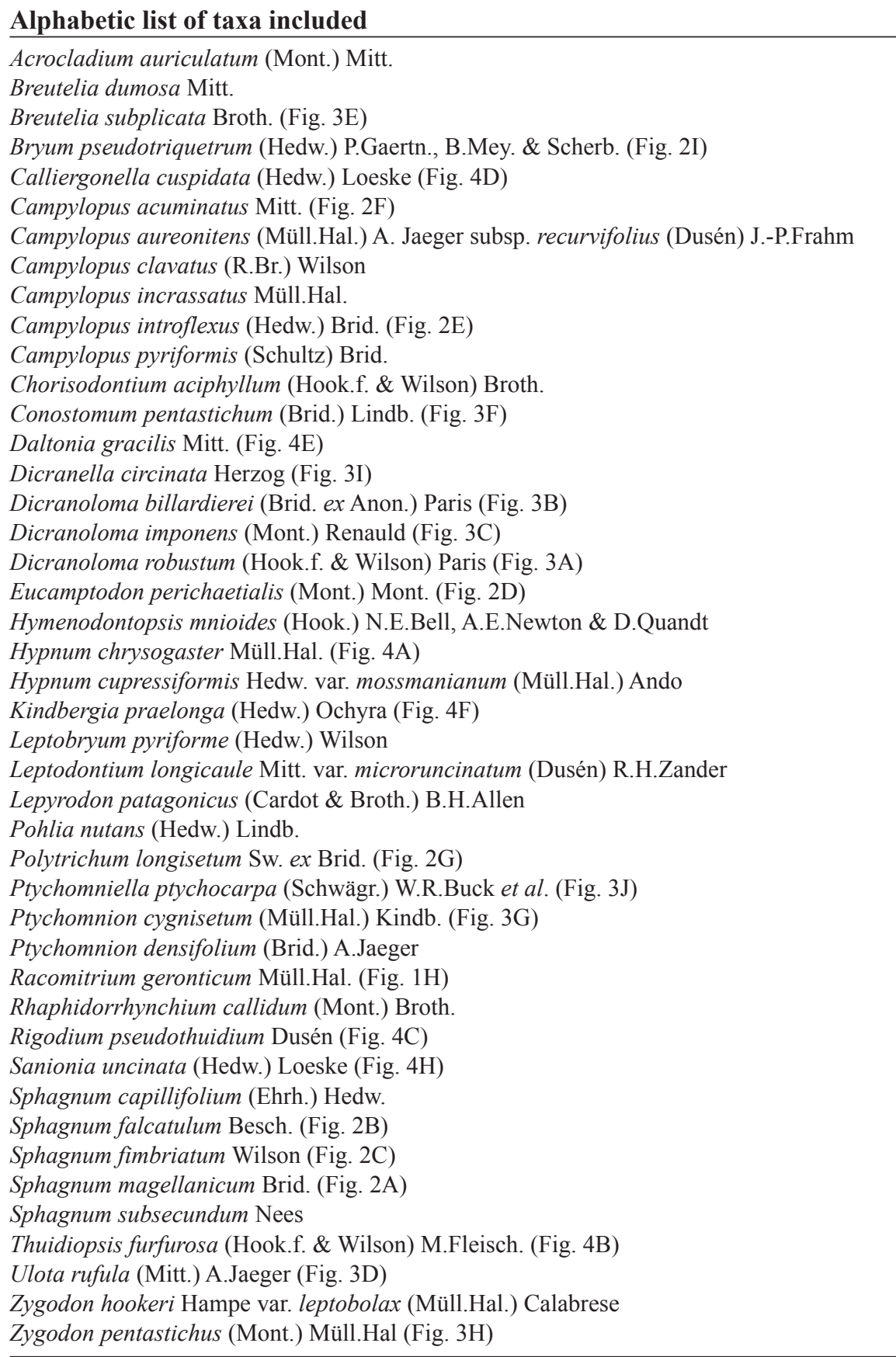



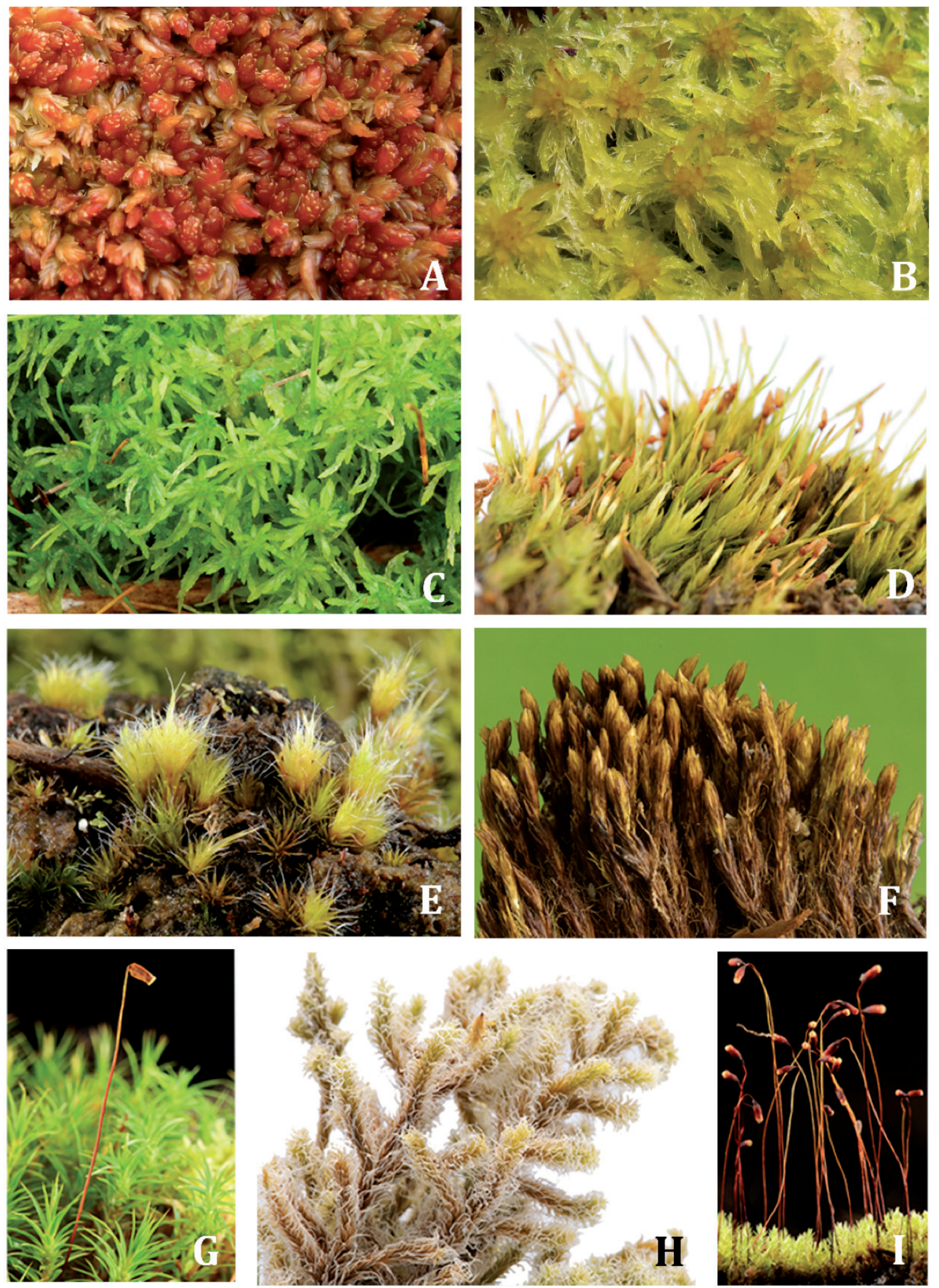

Figura 2. A, Sphagnum magellanicum Brid.; B, Sphagnum falcatulum Besch.; C, Sphagnum fimbriatum Wilson; D, Eucamptodon perichaetialis (Mont.) Mont.; E, Campylopus introflexus (Hedw.) Brid; F, Campylopus acuminatus Mitt.; G, Polytrichum longisetum Sw. ex Brid.; H, Racomitrium geronticum Müll.Hal.; I, Bryum pseudotriquetrum (Hedw.) P.Gaertn., B.Mey. \& Scherb. 

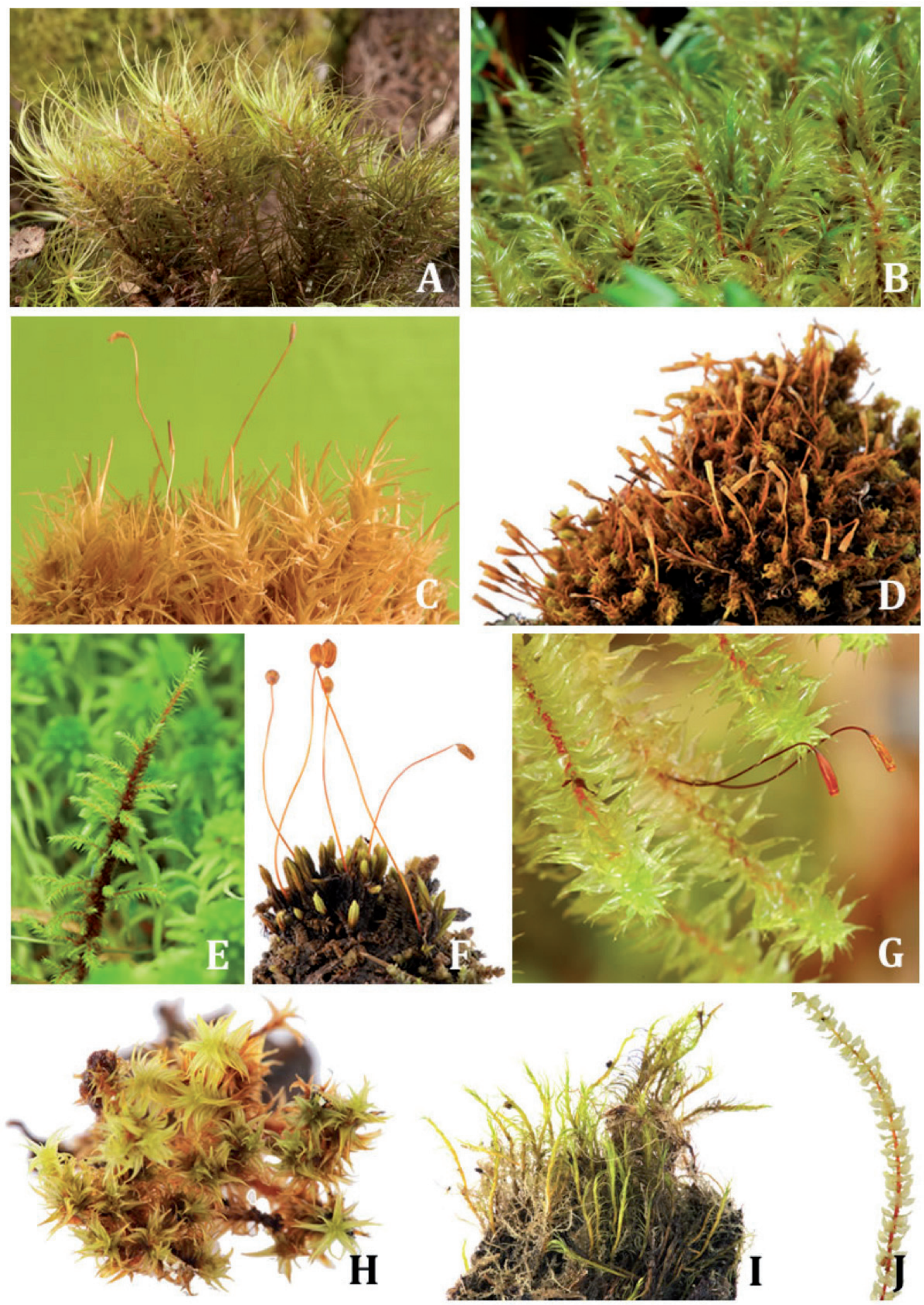

Figura 3. A, Dicranoloma robustum (Hook.f. \& Wilson) Paris; B, Dicranoloma billardierei (Brid. ex Anon.) Paris; C, Dicranoloma imponens (Mont.) Renauld; D, Ulota rufula (Mitt.) A.Jaeger; E, Breutelia subplicata Broth.; F, Conostomum pentastichum (Brid.) Lindb.; G, Ptychomnion cygnisetum (Müll.Hal.) Kindb.; H, Zygodon pentastichus (Mont.) Müll.Hal.; I, Dicranella circinata Herzog; J, Ptychomniella ptychocarpa (Schwägr.) W.R.Buck et al. 

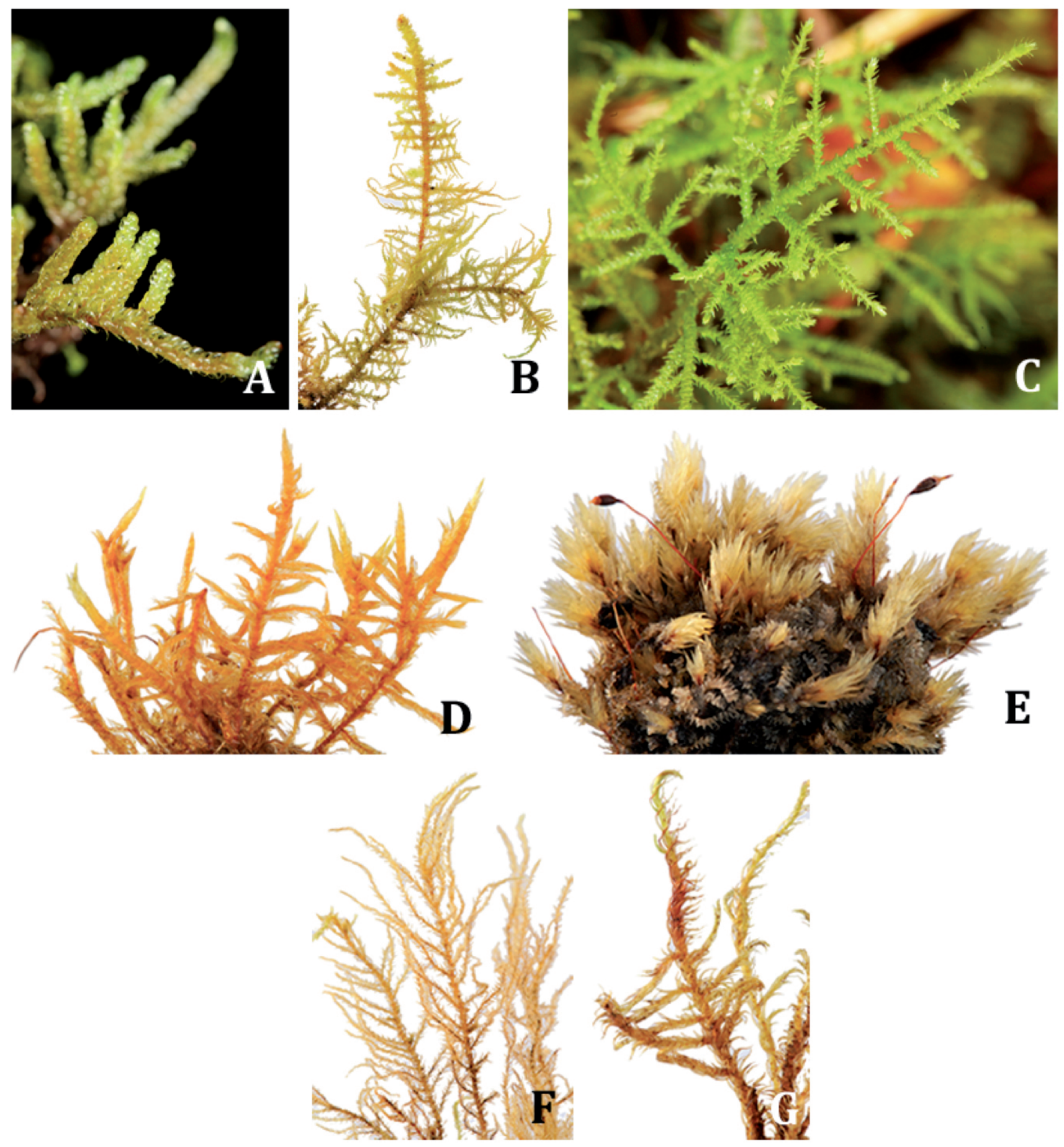

Figura 4. A, Hypnum chrysogaster Müll.Hal.; B, Thuidiopsis furfurosa (Hook.f. \& Wilson) M.Fleisch.; C, Rigodium pseudothuidium Dusén; D, Calliergonella cuspidata (Hedw.) Loeske; E, Daltonia gracilis Mitt.; F, Kindbergia praelonga (Hedw.) Ochyra, G, Sanionia uncinata (Hedw.) Loeske. 


\section{ACKNOWLEDGEMENTS}

This research was supported by FONDECYT 11150275, AECID A/025081/2009, Cooperación al Desarrollo UCM 4138114 and AECID A/030011/2011 grants. We are grateful to Alfonso Benítez-Mora for their field assistance and photographs. This is a contribution to the Research Program of LTSER-Chile network at Senda Darwin Biological Station, Chiloé, Chile.

\section{REFERENCES}

Buck, W.R., Goffinet, B. 2010. Preliminary key to the mosses of Isla Navarino, Chile (Prov. Antártica Chilena). Nova Hedwigia Beiheft 138: 215-229.

CONAF. 2009. Plan de Acción Provincial Chiloé - Plan de Gestión Territorial. Castro, Chile: Oficina Provincial Chiloé Corporación Nacional Forestal. 49 pp.
Di Castri, F., HajeK, E.R. 1976. Bioclimatología de Chile. Santiago, Chile: Editorial Universidad Católica de Chile. $128 \mathrm{pp}$.

LARRAín, J. 2007. Musgos (Bryophyta) de la estación biológica Senda Darwin, Ancud, isla de Chiloé: lista de especies y claves para su identificación. Chloris chilensis 10(1): http://www.chlorischile.cl/musgoschiloe/musgosfinal. htm.

Pérez, C. A., Armesto, J. J., Torrealba, C., Carmona, M.R. 2003. Litterfall dynamics and nitrogen use efficiency in two evergreen temperate rainforests of southern Chile. Austral Ecology 28(6): 591-600.

Robinson, H. 1975. The mosses of Juan Fernandez Islands. Smithsonian Contributions to Botany 27: 1-88.

VitT, D.H., Wieder, K. 2008. The structure and function of bryophyte-dominated peatlands. In B. Goffinet and A. J. Shaw (eds.), Bryophyte Biology, 357-391. Cambridge University Press.

Recibido: 28.01 .2018

Aceptado: 20.08 .2018 